\section{Fahr's disease: familial idiopathic basal ganglia calcification with and without extrapyramidal disorders}

\author{
Alberto Castagna, ${ }^{1}$ Carmen Ruberto, ${ }^{1}$ \\ Rosa Paola Cerra, ${ }^{2}$ Laura Greco, ${ }^{2}$ \\ Giovanni Ruotolo ${ }^{2}$ \\ ${ }^{1}$ Center for Cognitive Disorders and \\ Dementia, Azienda Sanitaria Provinciale \\ di Catanzaro; ${ }^{2}$ Geriatric Unit, \\ Pugliese-Ciaccio General Hospital, \\ Catanzaro, Italy
}

\begin{abstract}
Fahr's disease (FD), also known as familial idiopathic basal ganglia calcification, is a neurodegenerative disease affecting cerebral micro vessels, mainly in the basal ganglia. It mostly presents with movement disorders, dementia and behavioral abnormalities. It is considered hereditary with an autosomal dominant transmission. Fahr's disease is often underestimated and under diagnosed. We reported the clinical differences found in two patients with Fahr's Disease. In particular, we described a case of Fahr's disease with behavioral alteration with extrapyramidal movement disorders, and a rare case of Fahr's disease with cognitive and behavioral alterations in absence of extrapyramidal movement disorders.
\end{abstract}

\section{Introduction}

The primitive idiopathic calcification of the basal ganglia, also called Fahr's disease (FD), is a rare neurodegenerative disease of unknown etiology characterized by the presence of idiopathic, bilateral and symmetrical calcifications, at the level of the basal ganglia, the dentate nuclei of cerebellum, the thalamus nuclei and the semi-oval centre. ${ }^{1}$ Its prevalence increases with age and the most affected site is the globus pallidus. Symptoms and signs range from cases of asymptomatic patients to a progressive neuropsychiatric condition often associated with extrapyramidal movement disorders. These symptoms such as stiffness, hypokinesia, tremor, choreoathetosis, ataxia and buccal dyskinesia, are followed by cognitive disorders, then pyramidal signs, psychiatric disorders and changes in sensitivity and pain. ${ }^{2-4}$ Psychotic symptoms of Fahr's disease have the characteristics of secondary psychotic disorders and include auditory, olfactory and visual hallucinations, ideas of reference and influence, and persecutory delusional themes. ${ }^{5}$ Other neurological features are convulsions or events similar to stroke. ${ }^{2}$ Although dementia is a common disorder in $\mathrm{FD}$, the presentation of this disease with pure dementia (without extrapyramidal disorders) has been rarely reported. ${ }^{2,6}$ Treatment is symptomatic while the exact pathological process is unknown.

\section{Case Reports}

\section{Clinical case \#1 with extrapyramidal movement disorders}

A 81-year-old woman with hypertension and hypercholesterolemia, which were controlled with ramipril and atorvastatin, came to our observation for a progressive cognitive impairment started about 3 years earlier with movement disorders and occasional episodes of visual hallucinations, resistant to promazine and quetiapine therapy. The neurological clinical examination revealed that the patient had a pletora of symptoms most commonly movement disorders, cognitive impairment, and ataxia. Other neurologic manifestations included pyramidal signs (rigidity and bradykinesia), psychiatric features, and gait disorders. It was noted that she had intentional tremor and stooped posture. Routine blood chemistry tests, including calcium, phosphorus, as well as the hormonal profile, including thyroid hormones, parathyroid hormone and 25-OHVitamin D dosage were in normal ranges, the chest X-ray and ultrasound of the supraaortic vessels were normal as well. The brain CT showed: bilateral calcifications of several brain regions (Figure 1). The electroencephalogram (EEG) did not reveal any abnormalities. The Mini Mental State Examination corrected (MMSEc - Italian version), ${ }^{7}$ score was $15,4 / 30$ with marked deficit of the memory, Activities of Daily Living (ADL) score was 4/6; Instrumental Activities of Daily Living (IADL) 2/8; UCLA Neuropsychiatric Inventory (NPI) 24/144. ${ }^{8}$ The initial differential diagnoses entertained were Parkinson's disease and an ischemic stroke, but FD was diagnosed basing on exclusion of infective, toxic, traumatic causes and absence of biochemical abnormalities and somatic features suggestive of a mitochondrial or metabolic disease or other systemic disorder, progressive neuropsychiatric manifestations, bilateral calcifications of brain regions. The patient started therapy with levodopa and clonazepam in addition to the routine
Correspondence: Alberto Castagna, Center for Cognitive Disorders and Dementia DSS Catanzaro, Azienda Sanitaria Provinciale di Catanzaro, viale Crotone, 88100 Catanzaro, Italy.

Tel./Fax: +39.096.17033013

E-mail: albertocastagna@tiscali.it

Key words: Basal ganglia calcification; Fahr's disease; extrapyramidal disorders.

Conflict of interest: the authors declare no potential conflict of interest.

Received for publication: 9 November 2019. Revision received: 6 April 2020.

Accepted for publication: 6 April 2020.

This work is licensed under a Creative Commons Attribution-NonCommercial 4.0 International License (CC BY-NC 4.0).

${ }^{\circ}$ Copyright: the Author(s), 2020

Licensee PAGEPress, Italy

Geriatric Care 2020; 6:8670

doi:10.4081/gc.2020.8670

antihypertensive and lipid lowering drugs. Symptoms were persistent over a period of 5 weeks.

\section{Clinical case \#2 without extrapyramidal disorders}

A 73-year-old woman with hypercholesterolemia, came to our observation for a progressive cognitive impairment which started about 2 years earlier with occasional episodes of visual hallucinations and delusional ideation, changes in alertness, non-concrete reasoning, calculation, and occasional behavioral disturbances. General and neurological clinical examination was normal. Standard blood chemistry tests, including calcium, phosphorus, as well as the hormonal profile, including thyroid hormones and parathyroid hormone, were normal while the $25-\mathrm{OH}$ Vitamin D dosage was reduced to 11.4 $\mathrm{ng} / \mathrm{mL}$. Electrocardiogram (ECG) showed a type of atrial conduction block in first-degree, the chest X-ray and ultrasound of the supraaortic vessels were normal. The brain CT was normal for morphology and size the ventricular system with modest dilatation of the subarachnoid spaces of the base and of convexity on an atrophic basis, hypodensity of the periventricular white matter in relation to chronic vascular suffering, bilateral calcifications of lenticular nuclei, the corona radiata on the left and in the ipsilateral cerebellar site (Figure 2). Patient refused PET exam execution. Multidimensional assessment highlighted: MMSEc score was 19/30 with marked deficit of the memory and 
of the visuo-spatial abilities, ADL score was 5/6; UCLA NPI 12/144. FD was diagnosed basing on absence of biochemical abnormalities and somatic features suggestive of a mitochondrial or metabolic disease or other systemic disorder, progressive neuropsychiatric manifestations, bilateral calcifications of brain regions The patient started therapy with vitamin D3, citicoline $500 \mathrm{mg} \times 2$ /day, haloperidol $0.2 \% \quad 4$ drops/day, with frequency reduction of visual hallucinations episodes. At 18 months of follow-up. ${ }^{7-9}$ the MMSE totals a score of $9 / 30$, ADL are further compromised (ADL 1/6) and extrapyramidal disorders remain absent, even in the presence of a good control of visual hallucinations with haloperidol $0.2 \% 3$ drops $\times 3$ /day (NPI 2/144).

\section{Discussion}

Fahr's disease, described for the first time by Karl Theodor Fahr in 1930, is an unusual degenerative neurological disorder whose prevalence is probably $<0.5 \% .^{10}$ Diagnosis of Fahr's syndrome requires demonstration of etiological causes linked to the characteristic calcifications. According to our knowledge there are few cases described in the literature with same clinical features of the case reported above..$^{2,6,11-13}$ The first case of FD with pure and pre-senile dementia without extrapyramidal symptoms or metabolic abnormalities has been described in a patient with bilateral striopallido-dentate calcinosis and cortical atrophy prevalently in parietal-temporal areas with mild periventricular hyperintensity. ${ }^{6}$ Considering instrumental diagnosis, CT study, in some cases, can be integrated with a PET study. Benke et al. ${ }^{2}$ have shown, using PET studies in patients with FD and rapidly progressive dementia with predominant frontal lobe syndrome, a massive reduction in glucose metabolism of basal ganglia and in frontal lobe, related with the clinical condition of disinhibition and personality changes. F-Dopa striatal PET did not show nigro-striatal dopaminergic dysfunctions. Brain metabolism studied by Benke et al. can explain cognitive and behavioral problems in FD with dementia and with or without mild Parkinsonian symptoms. ${ }^{2}$ Unfortunately, our patient refused PET execution. Based on the age of onset and the two clinical patterns we described here two distinct clinical scenarios. The first case is characterized by an early onset, about 30 years, with schizophrenia psychosis in absence of movement disorders; the second one is characterized by late onset, about 50 years, in which progressive dementia and movement disorders prevail. The pathogenesis of cognitive disorders is linked to destruction of connections between ganglia and cortex, with subsequent frontotemporal atrophy, formation of neurofibrillary cortical aggregates and both cortical and basal neuronal loss. The psychotic symptoms of FD have characteristics of secondary psychotic disorders and include auditory, olfactory and visual hallucinations, ideas of reference and influence, and persecutory delusional themes. ${ }^{14}$ The most common symptoms of FD in adults include Parkinsonism, dystonia, ataxia, chorea and extrapyramidal syndromes. Some patients may present convulsions and pyramidal disorders. ${ }^{15}$ Currently, CT is the most valuable method that exceeds magnetic resonance imaging (MRI). CT results are important for a correct differential diagnosis with respect to frontotemporal dementia (FTD). ${ }^{16-18}$ If basal ganglia calcifications are secondary to a known cause, the disease is called Fahr's syndrome. ${ }^{19-21}$ This syndrome is associated with many alterations of calciumphosphorus metabolism disease (hyper-hypoparathyroidism,

pseudohypoparathyroidism), ${ }^{22,23} \mathrm{CNS}$ infections (AIDS, cytolomegalovirus), lupus systemic erythematosus but also to neoplasms such as angiomas and cerebral gliomas. In ours patients manifested PTH in the normal range. A possible association between Fahr's syndrome and head injuries ${ }^{24}$ and schizophrenia has also been reported ${ }^{25}$ Calcifications occur more often in elderly patients and may be symptoms of many diseases such as toxoplasmosis, cytomegalovirus, syphilis, tuberculosis, torulopsis, cysticercosis, tuberous sclerosis and others. ${ }^{26,27}$ It is worth mentioning that calcifications are quite commonly found in clinical practice and may be encountered as physiological calcifications in the pineal gland or choroid plexuses as well as on the walls of blood vessels. The deposits consist of calcium, zinc, iron, aluminium, magnesium, silicon, copper and phosphorus. $^{3}$ There are significant differences in the distribution of calcifications between individual patients. This may indicate the different mechanisms of their formation. The location within the brain structures as well as contact with blood

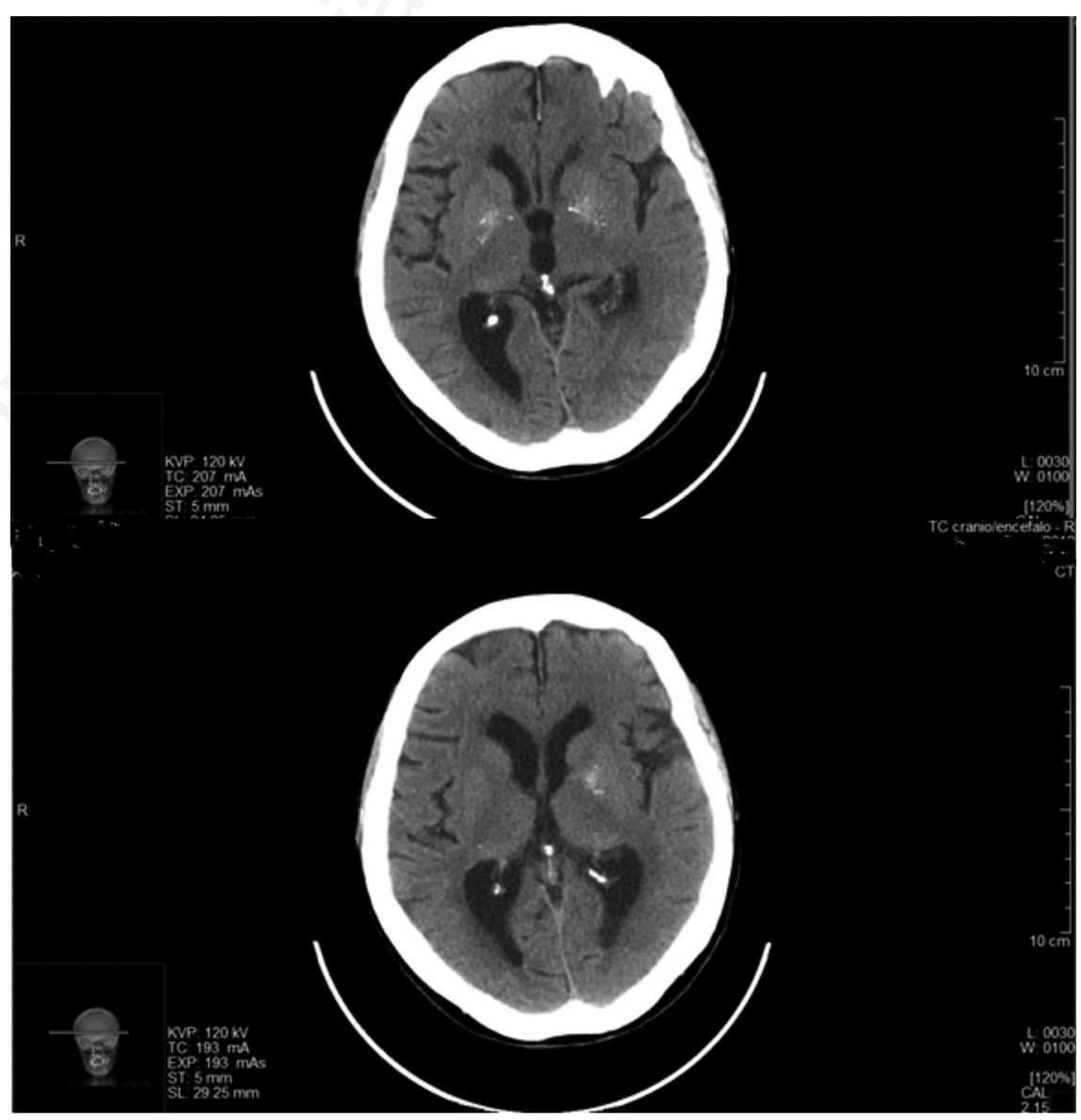

Figure 1. Brain computed tomography: bilateral calcification of brain regions. 
vessel are the most important factors determining the chemical composition of the deposits. On this basis, two types of mineralization were described, non-vascular and perivascular. FD pathophysiology is not yet known. Some reports describe the inheritance of Fahr's syndrome, mainly in an autosomal dominant way. ${ }^{26,27}$ Estimating prevalence is challenging due to the vast diversity of presenting symptoms and incomplete penetrance of the disease. However, with the most conservative estimations, the minimal prevalence of variants of known genes is $4.5 \mathrm{P}=10,000$ (95\%CI [3.4-5.5] $\mathrm{P}=10,000)$. Population genomic analysis reveals that this is not a very rare disease, and it has been underestimated and under diagnosed so far. ${ }^{28}$ Four genes have been proved to be related to primary familial brain calcification; namely, SLC20A2, PDGFRB, PDGFB and XPR1. However, in the majority of patients, the syndrome does not have a genetic background. There are some suggestions that intracerebral calcium deposition is secondary to local disturbance of the bloodbrain barrier or neuronal calcium metabolism disorder. ${ }^{29}$ An important argument in favor of vascular theory was given by studies using cerebral flow scintigraphy that provided evidence of altered blood flow to the calcification sites. ${ }^{30}$ Furthermore, inflammatory conditions, such as meningitis or encephalitis, are considered

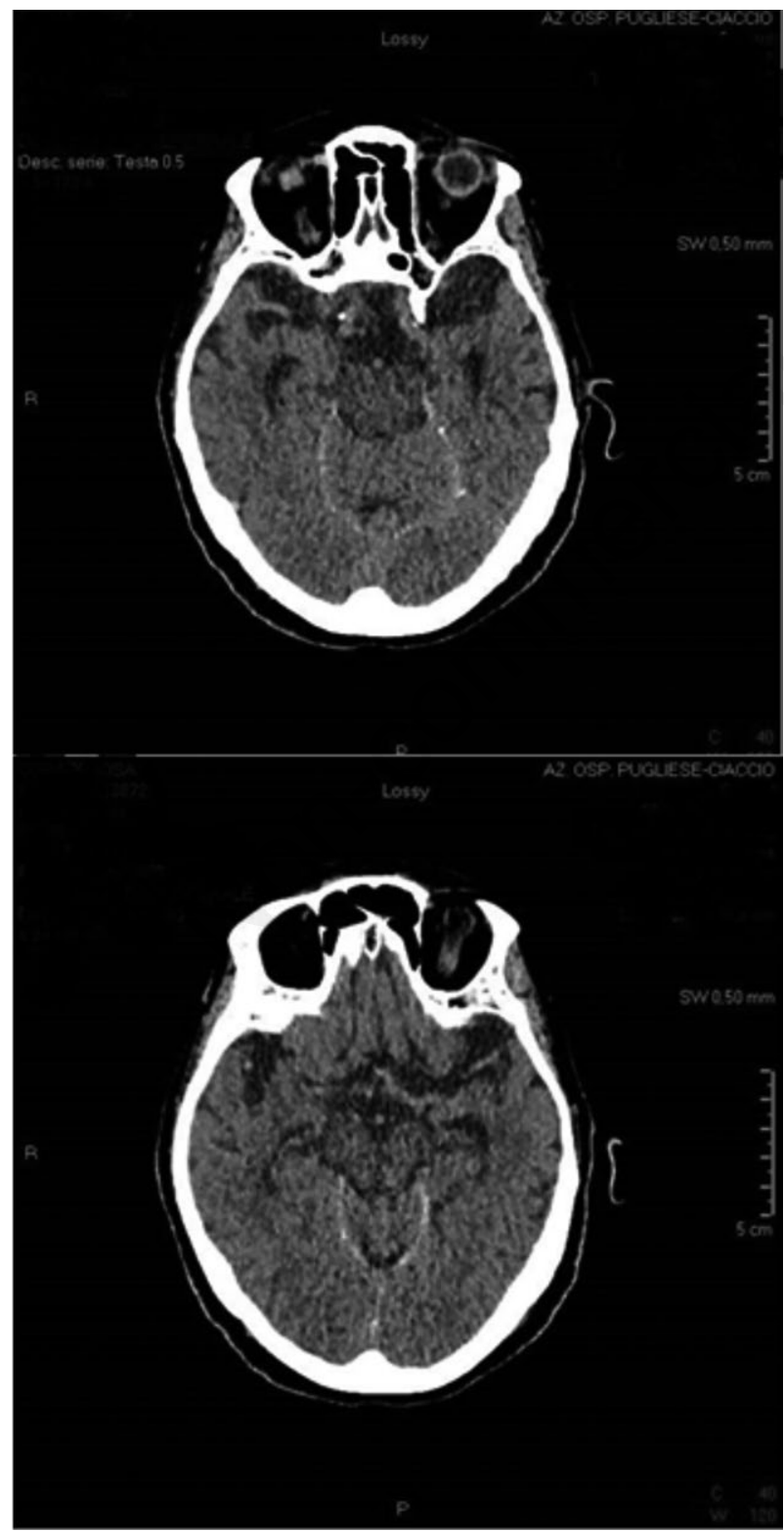

Figure 2. Brain computed tomography: bilateral calcification of lenticular nuclei and left corona radiata. in pathogenesis of Fahr's syndrome. ${ }^{31}$ There is no cure for FD, nor there is a standard course of treatment, which is limited to symptomatic therapy, considering that a peculiar characteristic of these patients is the poor response to neuroleptics with greater frequency of side effects and poor response to levodopa ${ }^{4}$ most probably due to the insensitivity of postsynaptic striatal structures rather than to the presynaptic damage observed in primary Parkinsonism. ${ }^{32}$

\section{Conclusions}

Fahr's disease is a neurodegenerative disorder presenting with a wide array of neuropsychiatric symptoms and is underestimated and under diagnosed. We reported the clinical differences found in two patients with FD. In particular, we described a case of FD with behavioral alteration with extrapyramidal movement disorders, and a rare case of FD with cognitive and behavioral alterations in absence of extrapyramidal movement disorders. Neurological and psychological examinations and $\mathrm{CT}$ imaging remain the basic techniques for the diagnosis of FD. Given the wide clinical variability of FD, the lack of specific treatments apart the symptomatic ones, it is helpful to keep in mind that a peculiar feature of these patients is the poor response to neuroleptics and the greater frequency of side effects ${ }^{4}$ along with to the poor response to levodopa. ${ }^{33}$

\section{References}

1. Manyam BV. What is and what is not 'Fahr's disease'. Parkinsonism Relat Disord 2005;11:73-80.

2. Benke T. Subacute dementia and imaging correlates in a case of Fahr's disease. J Neurol Neurosurg Psychiatry 2004;75:1163-5.

3. Lauterbach EC. Neuropsychiatric correlates and treatment of lenticulostriatal diseases: a review of the literature and overview of research opportunities in Huntington's, Wilson's, and Fahr's diseases. A report of the ANPA Committee on Research. American Neuropsychiatric Association. J Neuropsychiatry Clin Neurosci 1998;10:249-66.

4. Manyam BV, Walters AS, KR Narla KR. Bilateral striopallidodentate calcinosis: clinical characteristics of patients seen in a registry. Mov Disord 2001;16:258-64.

5. Cummings JL. Neuropsychiatric distur- 
bances associated with idiopathic calcification of the basal ganglia. Biol Psychiatry 1983;18:591-601.

6. Modrego PJ. Fahr's syndrome presenting with pure and progressive presenile dementia. Neurol Sci 2005;26:367-9.

7. Magni E, Binetti G, Bianchetti A, et al. Mini-Mental State Examination: a normative study in Italian elderly population. Eur J Neurol 1996;3:198-202.

8. Cummings JL. The Neuropsychiatric Inventory. Comprehensive assessment of psychopathology in dementia. Neurology 1994;44:2308.

9. Castagna A, Coppolino G, Leonardi G, et al. Fahr's disease: follow-up of a clinical case with severe cognitive impairment in absence of extrapyramidal disorders. J Gerontol Geriatr 2019;67:254-7.

10. Sgulo FG. Cerebrovascular disorders and Fahr's disease: Report of two cases and literature review. J Clin Neurosci 2018;50:163-4.

11. Weisman DC. Density of the brain, decline of the mind: an atypical case of Fahr disease. Arch Neurol 2007;64: 756-7.

12. Lam JS. Fahr's disease: a differential diagnosis of frontal lobe syndrome. Hong Kong Med J 2007;13:75-7.

13. Coppolino G, Bolignano D, Gareri P, et al. Kidney function and cognitive decline in frail elderly: two faces of the same coin? Int Urol Nephrol 2018;50:1505-10.

14. Shakibai SV, Johnson JP, Bourgeois JA. Paranoid delusions and cognitive impairment suggesting Fahr's disease. Psychosomatics 2005;46:569-72.

15. Hoque MA. Fahr's disease: a very rare cause of epilepsy. Mymensingh Med J 2010;19:127-9.

16 Sahin N. Fahr disease: use of susceptibility-weighted imaging for diagnostic dilemma with magnetic resonance imaging. Quant Imaging Med Surg 2015;5:628-32.

17. Shahidi GA, Safdarian M. Fahr disease: Idiopathic basal ganglia calcification. Iran J Neurol 2017;16:53-54.

18. Castagna A, Gareri P, Falvo F, et al. Werner syndrome:a rare mutation. Aging Clin Exp Res 2018;31:425-9.

19. Kiroglu Y. Intracranial calcifications on CT. Diagn Interv Radiol 2010; 16:263-9.

20. Batla A, Stamelou M. Primary familial brain calcification in the IBGC2 kindred: All linkage roads lead to SLC20A2. Mov Disord 2016;31:1765-6.

21. Bonazza S. Strio-pallido-dentate calcinosis: a diagnostic approach in adult patients. Neurol Sci 2011;32:537-45.

22. Stip E. Fahr's disease and Asperger's syndrome in a patient with primary hypoparathyroidism. J Neurol Neurosurg Psychiatry 2000;68:115-6.

23. Kalinowska-Nowak A. Calcifications of basal ganglia and cerebellum in patient with pseudohypoparathyroidism-case report. Przegl Lek 2002;59: 548-50.

24. Gurcan O. Chronic subdural hematoma associated with Fahr syndrome: a clinical association or just a simple coinci- dence? Asian J Neurosurg 2018;13:90-2.

25. Naqvi S. Fahr's syndrome misdiagnosed as schizophrenia: a case report. Cureus 2017;9:e1071.

26. Kotan D, Aygul R. Familial Fahr disease in a Turkish family. South Med J 2009; 102:85-6.

27. Wang H. Fahr's disease in two siblings in a family: a case report. Exp Ther Med 2015;9:1931-3.

28. Nicolas G. Estimation of minimal disease prevalence from population genomic data: Application to primary familial brain calcification. Am J Med Genet B Neuropsychiatr Genet 2018;177:68-74.

29. Pourshahid S. Fahr's Disease: A Differential to $\mathrm{Be}$ Considered for Various Neuropsychiatric Presentations. Cureus 2018;10:2304.

30. Uygur GA. Evaluation of regional cerebral blood flow in massive intracerebral calcifications. J Nucl Med 1995;36: 610-2.

31. Bobek J, Nowak M. Familial form of Fahr syndrome (report of two cases). Neurol Neurochir Pol 2000;34:167-75.

32. Klawans HL, Lupton M, Simon L. Calcification of the basal ganglia as a cause of levodopa-resistant Parkinsonism. Neurology 1976;26: 221-5.

33. Berendes K, Dorstelmann D. Unsuccessful treatment with levodopa of a Parkinsonian patient with calcification of the basal ganglia. J Neurol 1978;218:51-4. 